\title{
Rigorous Results Concerning Light Cone Dominance in Deep Inelastic Lepton-Hadron Scattering $\star$
}

\author{
Manfred Magg \\ Institut für Theoretische Physik, Technische Hochschule Aachen, \\ Aachen, Federal Republic of Germany
}

\begin{abstract}
We consider a class of distributions, appearing in electro-production and satisfying a Jost-Lehmann-Dyson representation. We then prove without further assumptions that only their singularity structure near the light cone is relevant for the behaviour of their Fourier transform in the Bjorken limit. Furthermore we study the ambiguity in this singularity structure introduced by the fact, that scaling is known only in the space like region of momentum space.
\end{abstract}

\section{Introduction ${ }^{1}$}

A great deal of theoretical interest has been shown in deep inelastic lepton-nucleon scattering experiments such as inelastic $e-p$ scattering. This has to do with the claim, often expressed in the literature, that in these experiments one is measuring the commutator of two currents in a region of the configuration space where the theoreticans may have some intuition, namely for light-like separations. This has led to the development of the so-called light cone physics [2]. However the arguments put forward as justification for the close relation between light cone and Bjorken limit are somewhat formal and not totally convincing [3].

In this paper we study rigorously the distribution theoretical problem of the dependence of the momentum space asymptotics on the configuration space structure of a structure function [4-6]. Because of the special singularity structure expressed by a Jost-Lehmann-Dyson representation it is possible to answer this question completely:

It is indeed the degree of singularity on the light cone which determines the Bjorken limit of structure functions (see our Theorem II).

This goes far beyond the general statements on asymptotic behaviour of Fourier transforms which can be found in standard books on distribution theory $[7,8]$.

* Work supported by the Bundesministerium für Forschung und Technologie.

1 This work is a slightly improved version of the author's thesis [1]. 
We further discuss the question of ambiguity in the light cone structure due to the fact that scaling can be measured for space-like momenta only. The result is the asymptotic analogue of the already known result concerning the arbitrariness in the determination of a causal distribution if one knows its spectrum in the space-like region only $[9,10]$.

\section{The Structure Functions of Inelastic $e-p$ Scattering and Their Bjorken Limit}

We recall the well known relation between the cross section for electroproduction ( $e+p \rightarrow e+$ anything) and the one-proton expectation value for the hadronic part of the electromagnetic current commutator [11]. The spinaveraged cross section depends on two invariant distributions $\hat{V}_{1}(q, p)$ and $\hat{V}_{2}(q, p)$ and has the form:

$$
\frac{d \sigma}{d \Omega d E^{\prime}}=\left(\frac{d \sigma}{d \Omega}\right)_{\text {Mott }}\left[-q^{2} \hat{V}_{2}+2 \tan ^{2} \frac{\theta}{2}\left(q^{2} \hat{V}_{1}+(p q)^{2} \hat{V}_{2}\right)\right]
$$

where $\theta$ is the scattering angle of the electron in the lab frame, $q$ its four momentum transfer and $E^{\prime}$ its final energy. The four momentum of the proton is denoted by $p$.

The so-called "causal structure functions" $\hat{V}_{1}$ and $\hat{V}_{2}$ are defined on the whole $q$-space by the following Fourier transform:

$$
\begin{aligned}
\frac{1}{2} \sum_{s= \pm \frac{1}{2}} \mathscr{F} & {\left[\left\langle p, s\left|\left[j_{\mu}^{h}(x), j_{v}^{h}(0)\right]_{t}\right| p, s\right\rangle\right](q) } \\
= & \left(q_{\mu} q_{\nu}-g_{\mu \nu} q^{2}\right) \hat{V}_{1}(q, p) \\
& +\left(\left(p_{\mu} q_{\nu}+p_{\nu} q_{\mu}\right) p q-p_{\mu} p_{\nu} q^{2}-g_{\mu \nu}(p q)^{2}\right) \hat{V}_{2}(q, p) .
\end{aligned}
$$

The subscript $t$ denotes the commutator without vacuum contribution.

The following properties of the "causal structure functions" are consequences of basic physical principles and will be relevant for our analysis $(i=1,2)$ :

i) For any fixed value of the four momentum $p$ contained in the mass shell

$$
\boldsymbol{M}:=\left\{p \in \mathbb{R}^{4} ; p^{2}=1, p_{0}>0\right\}
$$

$\hat{V}_{i}(q, p)$ is a tempered distribution in $q$-space.

Under a Lorentz transformation $L$ it behaves as follows:

$$
\left(\hat{V}_{i}(q, p), \varphi\left(L^{-1} q\right)\right)=\left(\hat{V}_{i}(q, L p), \varphi(q)\right) \quad\left(\varphi \in \mathscr{S}\left(\mathbb{R}^{4}\right)\right) .
$$


ii) For any fixed value of the parameter $p \in \boldsymbol{M} \hat{V}_{i}(q, p)$ is the Fourier transform of a causal distribution $V_{i}(x, p)$ :

$$
V_{i}(x, p)=0 \quad \text { for } \quad x^{2}<0
$$

iii) For any fixed value of the parameter $p \in \boldsymbol{M}$ the support of the distribution $\hat{V}_{i}(q, p)$ is contained in the following subset of the $q$-space:

$$
\left\{q \in \mathbb{R}^{4} ; q^{2}+2 p q \geqq 0 ; q_{0} \geqq 0\right\} \cup\left\{q \in \mathbb{R}^{4} ; q^{2}-2 p q \geqq 0, q_{0} \leqq 0\right\} \text {. }
$$

iv) $\hat{V}_{i}(-q, p)=-\hat{V}_{i}(q, p) \quad(p \in \boldsymbol{M})$.

All these properties are shown in the literature $[3,6,12]$.

Next we introduce the new real variables $\lambda, \omega$ and $n$. The fourvector $n$ obeys $n \cdot p=1$ and $n^{2}=0$. We define a linear coordinate transformation in the $q$-space by:

$$
q=\lambda n+\omega p
$$

The famous Bjorken scaling law can now be written in the following way $[10]$ :

$$
\begin{gathered}
\hat{V}_{1}(q, p)=\frac{F_{L}(\omega)}{\omega} \frac{1}{2 \lambda}+o\left(\lambda^{-1}\right) \\
\hat{V}_{2}(q, p)=\frac{F_{2}(\omega)}{\omega} \frac{1}{2 \lambda^{2}}+o\left(\lambda^{-2}\right) \\
\lambda \rightarrow+\infty, \quad \omega<0 .
\end{gathered}
$$

The one dimensional distribution $F_{L}$ and $F_{2}$ are called "scaling functions". The precise meaning of the Landau symbol $o(. .$.$) for distributions will$ be explained later on.

Remember that all experimental information about the structure functions is restricted to the region $q^{2}<0$, which has $\omega<0$ for $\lambda>0$.

The properties i)-iv) listed above define the class of tempered distributions which we want to study in the Bjorken limit. We continue to call the members of this class (generalized) structure functions (in momentum space).

Because of Lorentz covariance (property i) it is obviously sufficient to study these structure functions for the parameter value $p=(1,0,0,0)$ only (structure function in the "lab frame").

In the following we will restrict ourselves to this special choice and suppress the $p$-dependence.

For a structure function in $q$-space we shall write $\hat{T}$ or $\hat{T}(q)$. The corresponding distribution in configuration space will be denoted by $T$ or 
$T(x)$. Our convention for the Fourier transform is the following:

$$
\begin{gathered}
(\hat{T}(q), \varphi(q))=(T(x), \check{\varphi}(x)) \\
\check{\varphi}(x):=\int_{\mathbb{R}^{4}} d q e^{i q x} \varphi(q) \quad\left(\varphi \in \mathscr{S}\left(\mathbb{R}^{4}\right)\right) .
\end{gathered}
$$

The properties ii)-iv) are just the conditions which $T=V_{i}$ has to satisfy in order to have a Jost-Lehmann-Dyson representation [13]:

$$
T(x)=\int_{0}^{\infty} d s h(x, s) \Delta(x, s) .
$$

As usual $\Delta$ stands for the well known Pauli-Jordan function. The "spectral function" is a tempered distribution on $\mathbb{R}^{3} \times[0, \infty\rangle$ with the following properties:

Application of a test function $\varphi \in \mathscr{S}([0, \infty\rangle)$ in the variable $s$ gives a rotation invariant function $h_{\varphi}$ on $\mathbb{R}^{3}$

$$
h_{\varphi}(\boldsymbol{x}) ;=(h(\boldsymbol{x}, s), \varphi(s))_{s} .
$$

The spectrum of $h_{\varphi}$ (= support of the Fourier transform) is contained in some compact subset $\boldsymbol{K} \subset \mathbb{R}^{3}$. $\boldsymbol{K}$ is independent of $\varphi$.

One can specify the spectrum of $h_{\varphi}$ even further, but for our purpose this is sufficient.

One has to be a little bit careful in interpreting formula (2). In general it cannot be understood as an integral with respect to $d s$ even not in sense of distribution theory (unless $h$ is a function on $\mathbb{R}^{3} \times[0, \infty\rangle$ ). Its meaning is given by:

$$
\begin{aligned}
(T(x), \varphi(x)) & =(h(\boldsymbol{x}, s), \tilde{\varphi}(\boldsymbol{x}, s)) \\
\tilde{\varphi}(\boldsymbol{x}, s) & :=\int_{-\infty}^{+\infty} d x_{0} \Delta(x, s) \varphi\left(x_{0}, \boldsymbol{x}\right) .
\end{aligned}
$$

It is not difficult to see that $\tilde{\varphi}$ is a test function for the distribution $h$.

Instead of working with formula (2) we prefer a different representation for $T$ :

$$
T(x)=\int_{0}^{\infty} d s g(\boldsymbol{x}, s) \varepsilon\left(x_{0}\right) \delta^{l}\left(x^{2}-s\right) .
$$

The definition of $\varepsilon\left(x_{0}\right) \delta^{l}\left(x^{2}-s\right)(s \geqq 0, l=0,1,2, \ldots)$ is standard. The function $g$ is continuous, polynomially bounded on $\mathbb{R}^{3} \times[0, \infty\rangle$ and rotation invariant in the first variable. Its spectrum with respect to the first variable is contained in the same compact subset $\boldsymbol{K}$ as before. $\boldsymbol{K}$ is independent of $s \geqq 0$. The pair $(l, g)$ is then called a representation of the structure function $T$.

Formula (3) has the advantage that the $d s$-integral there, is a well defined parameter integral in the distribution sense [14]. We show in the 
appendix that a representation (3) can be derived from the Jost-LehmannDyson representation (2).

Formula (3) is the starting point of all our proofs.

There are several definitions in the literature for the asymptotic behaviour of distributions $[8,15]$. Schwartz seems to have given the most convenient one, both from the mathematical and the physical point of view. We apply it to the Bjorken limit.

Consider a distribution $U$ on $q$-space. The regularisation of $U$ by a function $\hat{\varphi} \in \mathscr{D}\left(\mathbb{R}^{4}\right)$ is a $C^{\infty}$-function. We can study the behaviour of this function in the limit:

$$
q=\lambda n+\omega p, \quad \lambda \rightarrow \infty .
$$

The symbol $p$ stands simply for $p=(1,0,0,0)$.

Definition. Let $U$ be a distribution on $q$-space, which is rotation invariant in the $q$ dependence.

Let $f:\langle 0, \infty\rangle \rightarrow\langle 0, \infty\rangle$ be a continous function.

We say: $U$ vanishes in the Bjorken limit faster than the function $f$, iff

$$
\lim _{\lambda \rightarrow \infty}(U * \hat{\varphi})(\lambda n+\omega p) \cdot(f(\lambda))^{-1}=0
$$

for any rotation invariant function $\hat{\varphi} \in \mathscr{D}\left(\mathbb{R}^{4}\right)$. For any fixed test function $\hat{\varphi}$ this limit is understood to be uniform with respect to $\omega, \omega$ varying in a compact intervall.

In symbols we write for this behaviour: $U(q) \underset{B}{\longrightarrow} o(f)$.

Obviously this definition does not depend on the special choice for the light like vector $n$. We demand the uniformness to fit the definition to the physical situation. The Landau symbol in (1) is now well explained.

\section{Light Cone Dominance}

In a first theorem we show that only the light cone in configuration space has a chance to contribute to the Bjorken limit of a structure function.

Theorem I. Let $T \in \mathscr{S}^{\prime}\left(\mathbb{R}^{4}\right)$ be as in formula (3). For an arbitrary $\varepsilon>0$ we have:

for all $n \in \mathbb{N}$.

$$
\mathscr{F}\left[\int_{\varepsilon}^{\infty} d s g(x, s) \varepsilon\left(x_{0}\right) \delta^{l}\left(x^{2}-s\right)\right](q) \underset{B}{\longrightarrow} o\left(\lambda^{-n}\right)
$$

Proof. We regularize the Fourier transform of

$$
T^{\varepsilon}(x):=\int_{\varepsilon}^{\infty} d s g(\boldsymbol{x}, S) \delta^{l}\left(x^{2}-s\right)
$$


through a test function with compact support. This is by the convolution theorem equivalent to the replacement of $g(\boldsymbol{x}, s)$ in the integral by its product with a function $\varphi \in \mathscr{F}\left[\mathscr{D}\left(\mathbb{R}^{4}\right)\right]$. The Fourier transform of this product

$$
\tilde{g}(q, s):=\int_{\mathbb{R}^{4}} d x e^{i q x} \varphi\left(x_{0}, \boldsymbol{x}\right) g(\boldsymbol{x}, s)
$$

has its support in the compact set:

$$
\tilde{\boldsymbol{K}}_{\varphi}:=\left\{(0, q)+q^{\prime} ; q \in \boldsymbol{K}, q^{\prime} \in \operatorname{supp} \hat{\varphi}\right\} .
$$

The regularized Fourier transform of $T^{\varepsilon}(x)$ is then given by:

$$
\hat{T}_{\varphi}^{\varepsilon}(q)=\int_{\varepsilon}^{\infty} d s\left(\tilde{g}(\cdot, s) *\left(-\frac{\partial}{\partial s}\right)^{l} \Delta(\cdot, s)\right)(q) .
$$

The star denotes the convolution with respect to the variable indicated by a dot. $\left(-\frac{\partial}{\partial s}\right)^{l} \Delta(q, s)$ is simply the Fourier transform of $\varepsilon\left(x_{0}\right) \delta^{l}\left(x^{2}-s\right)$, $(s \geqq 0)$. The convolution is well defined because $\tilde{g}(\cdot, s)$ is a function in $\mathscr{D}\left(\mathbb{R}^{4}\right)$ for any $s \geqq 0$.

We show now that the $d s$-integral converges. To this purpose we use an explicit form for $\left(-\frac{\partial}{\partial s}\right)^{l} \Delta(q, s)$ valid for $q_{0}>0$ and $s \geqq 0$ :

$$
\left(-\frac{\partial}{\partial s}\right)^{l} \Delta(q, s)=(-2)^{l}\left(\frac{1}{q_{0}} \frac{\partial}{\partial q_{0}}\right)^{k+1}\left[\left(q^{2}\right)_{+}^{\frac{l+k}{2}} s^{-\frac{l+k}{2}} J_{l+k}\left(\sqrt{s q^{2}}\right)\right] .
$$

$J_{l+k}$ is the Bessel function (of the first kind) of order $l+k$ and $\left(q^{2}\right)_{+}$ denotes the product of $q^{2}$ with the Heaviside function $\theta\left(q^{2}\right)$. The function within the squared bracket is analytic with respect to $s$ and $(l+k)$-times differentiable with respect to the $q$-variables. The formula is valid for $k=0,1,2, \ldots$. In the case $l=0$ the derivatives can be understood only in the sense of distribution theory. It is then a generalisation of a well known expression for the Pauli-Jordan function.

With this expression we can write the convolution integral as follows:

$$
\begin{aligned}
\left(\tilde{g}(\cdot, s) *\left(-\frac{\partial}{\partial s}\right)^{l} \Delta(\cdot, s)\right)(q) & (-2)^{l} s^{-\frac{l+k}{2}} \int_{\widetilde{\mathbf{K}}_{\varphi}} d q^{\prime}\left(\left(q-q^{\prime}\right)_{+}^{2}\right)^{\frac{l+k}{2}} J_{k+l}\left(\sqrt{s\left(q-q^{\prime}\right)^{2}}\right) \\
& \cdot\left(\frac{-\partial}{\partial q_{0}^{\prime}} \frac{1}{q_{0}-q_{0}^{\prime}}\right)^{K+1} \tilde{g}\left(q^{\prime}, s\right) .
\end{aligned}
$$


This equation is valid for those values of $q=\left(q_{0}, q\right)$ with

$$
q_{0}>\sup \left\{q_{0}^{\prime} \in \mathbb{R} ; q^{\prime} \in \tilde{\boldsymbol{K}}_{\varphi}\right\} .
$$

Now we substitute $q=\lambda n+\omega p$ in order to take the Bjorken limit. The scalar $\omega$ is contained in some compact intervall $\boldsymbol{J}$. There are the following bounds for the different factors in the integral above:

First, there exists a constant $C_{1}$ such that:

$$
\frac{\left(q-q^{\prime}\right)_{+}^{2}}{\lambda} \leqq C_{1} \quad\left(\lambda \geqq 1, q^{\prime} \in \tilde{\boldsymbol{K}}_{\varphi}\right) .
$$

The constant $C_{1}$ depends only on the two compact sets $\boldsymbol{J}$ and $\tilde{\boldsymbol{K}}_{\varphi}$.

Secondly, the absolute value of the Bessel function is bounded by 1 for all positive arguments.

Finally one verifies immediately the inequality:

$$
\left|\left(\frac{-\partial}{\partial q_{0}^{\prime}} \frac{1}{q_{0}-q_{0}^{\prime}}\right)^{k+1} \tilde{g}\left(q^{\prime}, s\right)\right| \leqq C_{2} \cdot \lambda^{-k-1}(1+s)^{m}
$$

with some integer $m$ and for all values of $\lambda>0$ such that:

$$
\lambda-1 \geqq \sup \left\{\left|q_{0}^{\prime}-\omega\right| ; \omega \in \boldsymbol{J}, q^{\prime} \in \tilde{\boldsymbol{K}}_{\varphi}\right\} .
$$

The result of these three properties is the bound:

$$
\begin{aligned}
\left|\left(\tilde{g}(\cdot, s) *\left(-\frac{\partial}{\partial s}\right)^{l} \Delta(\cdot, s)\right)(\lambda n+\omega p)\right| \\
\quad \leqq 2^{l} \frac{(1+s)^{m}}{s^{\frac{k+l}{2}}} \cdot \frac{\lambda^{\frac{l+k}{2}}}{\lambda^{k+1}} \cdot C_{1}^{\frac{l+k}{2}} \cdot C_{2} \cdot \int_{\widetilde{\mathbf{K}}_{\varphi}} d q^{\prime} .
\end{aligned}
$$

If we choose $k \in \mathbb{N}$ sufficiently large, this shows that the following integral is convergent:

$$
\hat{T}_{\varphi}^{\varepsilon}(\lambda n+\omega p)=\int_{\varepsilon}^{\infty} d s\left(\tilde{g}(\cdot, s) *\left(-\frac{\partial}{\partial s}\right)^{l} \Delta(\cdot, s)\right)(\lambda n+\omega p) .
$$

Furthermore we have proved the bound:

$$
\left|\hat{T}_{\varphi}^{\varepsilon}(\lambda n+\omega p)\right| \leqq 2^{l} \lambda^{\frac{l-k-2}{2}} C_{1}^{\frac{l+k}{2}} C_{2} \cdot \int_{\widetilde{\mathbf{K}}_{\varphi}} d q \cdot \int_{\varepsilon}^{\infty} d s \frac{(1+s)^{m}}{s^{\frac{k+l}{2}}}
$$

for $\lambda$ sufficiently large. Since $k \in \mathbb{N}$ may be chosen as large as we like this bound proves the theorem. 


\section{Singularity Structure on the Light Cone and Asymptotic Behaviour of the Fourier Transform}

In a second theorem we show the relation between the strength of the singularity on the light cone of a structure function and the behaviour of its Fourier transform in the Bjorken limit. This strength is characterized by $l$ the degree of singularity of $\varepsilon\left(x_{0}\right) \delta^{l}\left(x^{2}-s\right)$ with respect to $s$ and the Hölder index of the function $g(x, s)$ with respect to $s$ at the point $s=0$. The precise definition of this concept will be given later.

Theorem II: Let $T \in \mathscr{S}^{\prime}\left(\mathbb{R}^{4}\right)$ be as in formula (3). Furthermore if $\beta<1$ satisfies the two conditions:

a) $s^{\beta} g(\boldsymbol{x}, s)$ is polynomially bounded in $(\boldsymbol{x}, s)$ for $(\boldsymbol{x}, s) \in \mathbb{R}^{3} \times[0, \infty\rangle$.

b) $\lim _{s \rightarrow 0^{+}} s^{\beta} g(\boldsymbol{x}, s)=0$.

We then have the following bound for the Fourier transform $\hat{T}$ in the Bjorken limit:

$$
\hat{T} \underset{B}{\longrightarrow} O\left(\lambda^{l+\beta-2}\right) \text {. }
$$

Proof. According to Theorem 1 it is sufficient to prove the statement for the case $g(\boldsymbol{x}, s)=0$ for $s \geqq \varepsilon>0$. The representation (3) reduces in that case to:

$$
T(x)=\int_{0}^{\varepsilon} d s g(\boldsymbol{x}, s) \varepsilon\left(x_{0}\right) \delta^{l}(x-s) .
$$

By definition of the Bjorken limes we have to study the regularized Fourier transform $\hat{T}_{\varphi}$ of $T$.

The same arguments as in the proof before give:

$$
\begin{aligned}
& \hat{T}_{\varphi}(q)= \int_{0}^{\varepsilon} d s\left(\tilde{g}(\cdot, s) *\left(\frac{-\partial}{\partial s}\right)^{l} \Delta(\cdot, s)\right)(q) \\
&\left(\tilde{g}(\cdot, s) *\left(\frac{-\partial}{\partial s}\right)^{l} \Delta(\cdot, s)\right)(q) \\
&=(-2)^{l} s^{-\frac{l+k}{2}} \int_{\tilde{\boldsymbol{K}}_{\varphi}} d q^{\prime}\left(\left(q-q^{\prime}\right)_{+}^{2}\right)^{\frac{l+k}{2}} \\
& J_{l+k}\left(\sqrt{s\left(q-q^{\prime}\right)^{2}}\right)\left(\frac{-\partial}{\partial q_{0}^{\prime}} \frac{1}{q_{0}-q_{0}^{\prime}}\right)^{k+1} \tilde{g}\left(q^{\prime}, s\right)
\end{aligned}
$$

for

$$
q_{0}>\sup \left\{q_{0}^{\prime} \in \mathbb{R} ; q^{\prime} \in \tilde{\boldsymbol{K}}_{\varphi}\right\} \text {. }
$$

Remember $\tilde{g}$ was simply the following test function on $q$-space:

$$
\tilde{g}(q, s)=\int_{\mathbb{R}^{4}} d x e^{i q x} \varphi\left(x_{0}, \boldsymbol{x}\right) g(\boldsymbol{x}, s), \quad\left(\hat{\varphi} \in \mathscr{D}\left(\mathbb{R}^{4}\right)\right) .
$$


Let $P$ be an arbitrary polynomial depending on four variables. We have:

$$
P\left(-i \frac{\partial}{\partial q^{\mu}}\right) s^{\beta} \tilde{g}(q, s)=\int_{\mathbb{R}^{4}} d x e^{i q x} g(\boldsymbol{x}, s) s^{\beta} \varphi\left(x_{0}, x\right) P\left(x^{\mu}\right) .
$$

It follows immediately from our assumption a) that $\left|P\left(-i \frac{\partial}{\partial q_{\mu}}\right) s^{\beta} \tilde{g}(q, s)\right|$ is bounded for all $(q, s) \in \mathbb{R}^{4} \times[0, \infty\rangle$. If we use assumptions a) and b) and Lebesque's "Dominated Convergence Theorem" we conclude furthermore:

$$
\lim _{s \rightarrow 0^{+}} P\left(-i \frac{\partial}{\partial q_{\mu}}\right) s^{\beta} \tilde{g}(q, s)=0 \quad\left(q \in \mathbb{R}^{4}\right) .
$$

Now we investigate the Bjorken limit of $\hat{T}_{\varphi}(q)$ :

$$
\begin{gathered}
q=\lambda n+\omega p \\
\lambda \rightarrow \infty, \quad \omega \in \boldsymbol{J} \text { fixed. }
\end{gathered}
$$

$\boldsymbol{J}$ is a compact intervall.

For this purpose we substitute $u:=\lambda s$ in the integral expression for $\hat{T}_{\varphi}(q)$ and we derive for all values of $q$ with $q_{0}>\sup \left\{q_{0}^{\prime} \in \mathbb{R} ; q^{\prime} \in \tilde{\boldsymbol{K}}_{\varphi}\right\}$

$$
\begin{gathered}
\hat{T}_{\varphi}(q)=(-2)^{l} \cdot \lambda^{l+\beta-2} \int_{0}^{\varepsilon \lambda} d u u^{-\frac{l+k+2 \beta}{2}} \\
\int_{\widehat{\mathbf{K}}_{\varphi}} d q^{\prime}\left(\frac{\left(q-q^{\prime}\right)_{+}^{2}}{\lambda}\right)^{\frac{l+k}{2}} J_{k+l}\left(\sqrt{u \frac{\left(q-q^{\prime}\right)^{2}}{\lambda}}\right) \\
\cdot\left(\frac{-\partial}{\partial q_{0}^{\prime}} \frac{\lambda}{q_{0}-q_{0}^{\prime}}\right)^{k+1} \tilde{g}\left(q^{\prime}, \frac{u}{\lambda}\right)\left(\frac{u}{\lambda}\right)^{\beta} .
\end{gathered}
$$

In order to obtain a bound for that integral we use the following inequalities:

For the Bessel function $J_{k+l}$ one has the bound

$$
\left|J_{k+l}(\sqrt{y})\right| \leqq C_{k+l}^{\prime}\left(\frac{y}{y+1}\right)^{\frac{k+l}{2}}, \quad(y \geqq 0) .
$$

As mentioned in the proof of Theorem 1 we have

$$
\frac{\left(q-q^{\prime}\right)_{+}^{2}}{\lambda} \leqq C_{1}
$$

for those values of $q=\lambda n+\omega \varrho$ with $\lambda>1$ and $\omega \in \boldsymbol{J}$. The constant $C_{1}$ depends only on the two compacts sets $\tilde{\boldsymbol{K}}_{\varphi}$ and $\boldsymbol{J}$. 
For all those values of $q=\lambda n+\omega p$ with

$$
\lambda-1 \geqq \sup \left\{\left|q_{0}^{\prime}-\omega\right| ; \omega \in \boldsymbol{J}, q^{\prime} \in \tilde{\boldsymbol{K}}_{\varphi}\right\}
$$

it is easy to see that there exists a constant $C_{3, k}$ with

$$
\left|\left(\frac{\partial}{\partial q_{0}^{\prime}}\right)^{r}\left(\frac{\lambda}{q_{0}-q_{0}^{\prime}}\right)^{s}\right| \leqq C_{3, k}
$$

for all $r$ with $r \leqq s \leqq k+1$.

Combining these three inequalities we derive a bound for $\left|\hat{T}_{\varphi}(q)\right|$ :

$$
\begin{aligned}
2^{-l}\left|\hat{T}_{\varphi}(q)\right| & \lambda^{-l-\beta+2} \\
\leqq & C_{1}^{\frac{l+k}{2}} C_{k+l}^{\prime} C_{3, k} \int_{0}^{\infty} d u u^{-\frac{l+k+2 \beta}{2}} \\
& \cdot\left(\frac{u C_{1}}{1+u C_{1}}\right)^{\frac{k+l}{2}} \int_{\tilde{\boldsymbol{\kappa}}_{\varphi}} d q^{\prime}\left|P_{k}\left(\frac{\partial}{\partial q_{0}^{\prime}}\right) \tilde{g}\left(q^{\prime}, \frac{u}{\lambda}\right)\left(\frac{u}{\lambda}\right)^{\beta}\right| .
\end{aligned}
$$

This bound is valid for:

$$
\lambda-1 \geqq \sup \left\{\left|q_{0}^{\prime}-\omega\right| ; \omega \in \boldsymbol{J}, q^{\prime} \in \tilde{\boldsymbol{K}}_{\varphi}\right\} .
$$

$P_{k}$ denotes a one dimensional polynomial.

Remember $P_{k}\left(\frac{\partial}{\partial q_{0}^{\prime}}\right) \tilde{g}\left(q^{\prime}, \frac{u}{\lambda}\right)\left(\frac{u}{\lambda}\right)^{\beta}$ is bounded too. If we choose $k \in \mathbb{N}$ large enough to have $\lambda+k+2 \beta>2$ then the following integral

$$
\int_{0}^{\infty} d u u^{-\frac{l+k+2 \beta}{2}}\left(\frac{u C_{1}}{1+u C_{1}}\right)^{\frac{k+l}{2}} \int_{\widetilde{\mathbf{K}}_{\varphi}} d q^{\prime}, \text { const }
$$

converges (because of $\beta<1$ ). The integrand serves as a dominating function to apply Lebesque's "Dominated Convergence Theorem" to $2^{-l}\left|T_{\varphi}(q)\right| \lambda^{-l-\beta+2}$. It tells us that the right side of the inequality for $2^{-l}\left|T_{\varphi}(q)\right| \lambda^{-l-\beta+2}$ vanishes in the limit $\lambda \rightarrow \infty$ because $P_{k}\left(\frac{\partial}{\partial q_{0}^{\prime}}\right) \tilde{g}\left(q^{\prime}, \frac{u}{\lambda}\right)\left(\frac{u}{\lambda}\right)^{\beta}$ vanishes there, for $u>0$.

The constants $C_{1}, C_{k+l}^{\prime}$ and $C_{3, k}$ do not depend on the particular value of $\omega \in \boldsymbol{J}$. The convergence is therefore uniform with respect to $\omega \in \boldsymbol{J}$. This completes the proof.

Remark. The function $g$ in formula (3) is continuous in $\mathbb{R}^{3} \times[0, \infty\rangle$. Therefore any $\beta>0$ satisfies the assumptions a) and b) in Theorem II. The condition $\beta<1$ is therefore no restriction.

The rotation invariance of $g(\boldsymbol{x}, s)$ is not used in the proofs of theorem I and theorem II. These theorems are true without this assumption. This fact may be helpful if one wants to study the Bjorken asymptotics for non forward Compton scattering. 


\section{The Degree of Singularity on the Light Cone}

Here we give a precise definition for the degree of the light cone singularity of a structure function $T$ [16]. First we note that the integer $l$ and the function $g$ in formula (3) are not uniquely determined by the structure function $T$. If $(l, g)$ defines a representation (3) of $T$, it is obviously true for $\left(l+1, g^{(-1)}\right)$ too, where

$$
g^{(-1)}(\boldsymbol{x}, s):=\int_{0}^{s} d s^{\prime} g\left(\boldsymbol{x}, s^{\prime}\right)
$$

Of course we would like this degree of singularity to have a meaning independent of the special representation. This is done by the following definition:

Definition. Let $T$ be a generalized structure function in configuration space. Consider the infimum of all numbers $l+\beta$ such that there is a representation $(l, g)$ of $T$ and $s^{\beta} g(\boldsymbol{x}, s)$ is polynomially bounded in $(\boldsymbol{x}, s)$ for all $(\boldsymbol{x}, s) \in \mathbb{R}^{3} \times[0, s\rangle$.

Then this infimum is referred to as the degree of singularity of $T$ on the light cone. We denote it by SG $[T]$. Note that $-\infty$ is not excluded.

To justify this definition we mention the following properties of SG:

i) We have $\mathrm{SG}[T]=-\infty$ if the structure function coincides with a polynomially bounded $C^{\infty}$-function in some neighbourhood of the light cone. This is true especially for those $T$ with

$$
\operatorname{supp} T \subset\left\{x \in \mathbb{R}^{4}, x^{2}>\varepsilon\right\} .
$$

ii) We have $\mathrm{SG}[T]=-\sigma$ for $T(x)=f(x) \operatorname{disc}\left(x^{2}\right)^{\sigma} . f$ is a function with compact spectrum and $\operatorname{disc}\left(x^{2}\right)^{\sigma}$ is a shorthand for:

$$
\Gamma(-\sigma)\left[\left(-x^{2}+i \varepsilon x_{0}\right)^{\sigma}-\left(-x^{2}-i \varepsilon x_{0}\right)^{\sigma}\right], \quad(\sigma \in \mathbb{R}) .
$$

Now we are ready for our first important application to inelastic $e-p$ scattering:

It is an immediate consequence for our Theorem II that the scaling relations (1) can hold only if the structure functions $V_{1}$ and $V_{2}$ have:

$$
\mathrm{SG}\left[V_{1}\right] \geqq 1, \quad \mathrm{SG}\left[V_{2}\right] \geqq 0 .
$$

Here we assume that the scaling functions do not vanish identically.

Proof. If the inequalities (4) would not hold, then there is a representation (3) for $V_{1}$ given by $(l, g)$ and a $\beta$ such that Theorem II applies with $l+\beta<1$. Consequently we have

$$
\hat{V}_{1} \underset{B}{\longrightarrow} O\left(\lambda^{\varrho}\right)
$$

with $\varrho<-1$. This is a contradiction to (1) unless $F_{L}$ vanishes identically. The same argument with $\varrho<-2$ applies to $\hat{V}_{2}$ and $F_{2}$. 
The concept of the degree of singularity on the light cone makes it possible to define a light cone expansion [17].

Definition. An expansion of a structure function $T$ into a formal series $\sum_{i=1}^{\infty} T_{i}$ of structure functions is called a light cone expansion of $T$ iff the sequence of the remainders has strictly decreasing singularity degrees on the light cone.

As a consequence of Theorem II, we are allowed to Fourier transform a light cone expansion term by term and the result is an asymptotic expansion of the Fourier transform in the Bjorken limit.

In the preceeding paragraph we found a lower bound for $\operatorname{SG}\left[V_{1}\right]$ and $\mathrm{SG}\left[V_{2}\right]$ as a consequence of the experimentally verified scaling law. It is well known that it is impossible to derive an upper bound for these degrees from the scaling law (1). One is always free to add to $V_{1}$ or $V_{2}$ terms like: $[10,18]$

$$
P\left(x_{0}\right) \chi(x)
$$

$P$ denotes a polynomial and $\chi$ is a Lorentz invariant, uneven distribution. The Fourier transform of this distribution vanishes in the measurable region $q^{2}<0$ and satisfies the Conditions I)-IV) for structure functions. Since $\chi$ can be chosen as singular as one likes, this shows the impossibility to derive an upper bound for $\mathrm{SG}\left[V_{i}\right]$ as a consequence of the relation (1) only.

We are going to demonstrate that singularities with $\mathrm{SG}>1$ (resp. $\mathrm{SG}>0$ ) in the structure function $V_{1}$ (resp. $V_{2}$ ) are necessarily of the type $P\left(x_{0}\right) \chi(x)$ mentioned before.

Take $V_{1}$ for example. We assume that $V_{1}$ has a light cone expansion as follows:

$$
V_{1}(x)=\left.\sum_{i=0}^{N} \sum_{j=0}^{n_{i}} f_{i, j}\left(x_{0}\right)\left(\frac{\partial}{\partial \sigma}\right)^{j} \operatorname{disc}\left(x^{2}\right)^{\sigma}\right|_{\sigma=\sigma_{\imath}}+T_{N}(x) .
$$

The defining condition is thereby $\sigma_{i}<\sigma_{i+1} \leqq-1$, SG $\left[T_{N}\right]<1$. Logarithmic correction terms are taken into account by differentiating $\operatorname{disc}\left(x^{2}\right)^{\sigma}$ with respect to the exponent $\sigma \in \mathbb{R}$.

The Fourier transform of a term:

$$
f\left(x_{0}\right)\left(\frac{\partial}{\partial \sigma}\right)^{j} \operatorname{disc}\left(x^{2}\right)^{\sigma}
$$

is in Bjorken limit equal to:

$$
-\pi^{2}\left(\frac{\partial}{\partial \sigma}\right)^{j}\left[2^{2 \sigma+4}(2 \lambda)^{-\sigma-2} \cdot\left(\hat{f} * g_{-\sigma-2}\right)(\omega)\right]+o\left(\lambda^{-\sigma-2}\right) .
$$


Here we introduced:

$$
g_{\sigma}(y):=(\Gamma(\sigma+1))^{-1} y_{+}^{\sigma} \quad(\sigma \in \mathbb{R})
$$

and we made the substitution $q=\lambda n+\omega p$. From this expression and Theorem II we obtain for the Fourier transform $\hat{V}_{1}$ in the Bjorken limit:

$$
\begin{aligned}
\hat{V}_{1}(\lambda n+\omega p)= & -\pi^{2}\left(\frac{\lambda}{1}\right)^{-\sigma_{0}-2}(-\ln 2 \lambda)^{n_{0}} \cdot\left(\hat{f}_{0,0} * g_{-\sigma_{0}-2}\right)(\omega) \\
& +o\left(\lambda^{-\sigma_{0}-2} \cdot(\ln \lambda)^{n_{0}}\right) .
\end{aligned}
$$

Comparing this result with the scaling law (1), we conclude that the coefficient $\left(f_{0,0} * g_{-\sigma_{0}-2}\right)(\omega)$ in the expansion (6) has to vanish for $\omega<0$.

Since supp $\hat{f}_{0,0}$ is compact and symmetric, its convex hull is given by an intervall $[-a, a], a \geqq 0$. The support of $g_{-\sigma-2}$ is either the whole intervall $[0, \infty\rangle$ or concentrated at the origin (for $\sigma_{0}=-1,0,1, \ldots$ ). We write:

with:

$$
g_{-\sigma_{0}-2}=\tilde{g}_{-\sigma_{0}-2}+\tilde{\tilde{g}}_{-\sigma_{0}-2}
$$

$$
\operatorname{supp} \tilde{\tilde{g}}_{-\sigma_{0}-2} \subset[1+a, \infty\rangle
$$

and:

$$
\operatorname{supp} \tilde{g}_{-\sigma_{0}-2} \subset[0,2+a] \text {. }
$$

The vanishing of $\hat{f}_{0,0} * g_{-\sigma_{0}-2}$ on the left side of the real axis is obviously equivalent to the vanishing of $\hat{f}_{0,0} * \tilde{g}_{-\sigma_{0}-2}$ for this set. The convex hull of $\operatorname{supp} \tilde{g}_{-\sigma_{0}-2}$ is given by an intervall $[0, b]$ with $0 \leqq b \leqq 2+a$.

An important theorem of Titchmarsh on the support of a convolution gives the relation [19]:

$$
[-a, a]+[0, b] \subset[0, \infty>\text {. }
$$

This can be true only for $a=0$. The support of $\hat{f}_{0,0}$ is therefore concentrated at the origin. According to a well known theorem in distribution theory this forces $f_{0,0}$ to be a polynomial. Consequently the spectrum of $f_{0,0}\left(x_{0}\right)\left(\frac{\partial}{\partial \sigma}\right)^{n_{0}} \operatorname{disc}\left(x^{2}\right)^{\sigma}$ contains no space like momenta and its Fourier transform does not contribute in the measurable region.

We can repeat now the same argument with the next, less singular term in the expansion (5) and so on.

In this way we derive that all $f_{i, j}$ with $i \leqq N-1$ or $i=N$ but $j>0$ have to be polynomials. Only for $(i, j)=(N, 0)$ we must have $\sigma_{N}=-1$ and we get the well known relation for the scaling function:

$$
-\frac{\pi^{2}}{2}\left(\hat{f}_{N, 0} * g_{-1}\right)(\omega)=\frac{F_{L}(\omega)}{\omega} \quad(\omega<0) .
$$


This can be written also in the form:

$$
4 \pi^{2} \hat{f}_{N, 0}(\omega)=\frac{F_{L}(\omega)}{\omega} \quad(\omega<0) .
$$

The same analysis applies to $V_{2}(q)$ and $F_{2}(\omega)$, if we replace $\sigma_{N}=-1$ by $\sigma_{N}=-2$. Therefore the higher than canonical singular terms in an expansion of type (4) have to be of the already mentioned kind $P\left(x_{0}\right) \chi(x)$.

Acknowledgements. I would like to thank Professor H. A. Kastrup for many helpful discussions and his continuous interest in this work.

\section{Appendix}

We start with a J.L.D.-representation for $T$ :

$$
T(x)=\int_{0}^{\infty} d s h(\boldsymbol{x}, s) \Delta(x, s) .
$$

For any test function $\psi \in \mathscr{S}([0, \infty\rangle)$ is

$$
\boldsymbol{x} \sim(h(\boldsymbol{x}, s), \psi(s))_{s}
$$

an entire function with compact spectrum $\boldsymbol{K}_{\psi}$ and there exists a compact set $\boldsymbol{K} \subset \mathbb{R}^{3}$ such that:

$$
\boldsymbol{K}_{\psi} \subset \boldsymbol{K} \quad(\psi \in \mathscr{S}([0, \infty\rangle)) .
$$

The J.L.D.-representation contains more information on the spectra of these functions, but we shall not use it.

If $\varphi \in \mathscr{S}\left(\mathbb{R}^{4}\right)$, we have

with:

$$
(T(x), \varphi(x))=(h(\boldsymbol{x}, s), \tilde{\varphi}(\boldsymbol{x}, s))
$$

$$
\tilde{\varphi}(\boldsymbol{x}, s):=\int_{-\infty}^{+\infty} d x_{0} \Delta(x, s) \varphi\left(x_{0}, \boldsymbol{x}\right), \quad\left(\boldsymbol{x} \in \mathbb{R}^{3}, s \geqq 0\right) .
$$

For the Pauli-Jordan function we have the following expression:

$$
\begin{aligned}
\Delta(x, s) & =\varepsilon\left(x_{0}\right)\left[\frac{1}{x_{0}} \frac{\partial}{\partial x_{0}} \theta\left(x^{2}\right) J_{0}\left(\sqrt{s x^{2}}\right)\right] \\
& =\varepsilon\left(x_{0}\right)\left[\left(\frac{1}{x_{0}} \frac{\partial}{\partial x_{0}}\right)^{k+1}\left(\left(x^{2}\right)_{+}^{k} \frac{J_{k}\left(\sqrt{s x^{2}}\right)}{\left(\sqrt{s x^{2}}\right)^{k}}\right)\right]
\end{aligned}
$$

for $k=0,1,2, \ldots$. 
With a test function $\varphi \in \mathscr{S}\left(\mathbb{R}^{4}\right)$ we obtain:

$\left(\Delta(x, s), \varphi\left(x_{0}, \boldsymbol{x}\right)\right)_{x_{0}}$

$$
=2 \int_{0}^{\infty} d x_{0}\left(x^{2}\right)_{+}^{k} \frac{J_{k}\left(\sqrt{s x^{2}}\right)}{\left(\sqrt{s x^{2}}\right)^{k}}\left(\frac{-\partial}{\partial x_{0}} \frac{1}{x_{0}}\right)^{k+1} \frac{\varphi\left(x_{0}, \boldsymbol{x}\right)-\varphi\left(-x_{0}, \boldsymbol{x}\right)}{2} .
$$

We introduce the abbreviation:

$$
\chi_{k}\left(x_{0}, \boldsymbol{x}\right):=\left(\frac{-1}{x_{0}} \frac{\partial}{\partial x_{0}}\right)^{k+1} \frac{\varphi\left(x_{0}, \boldsymbol{x}\right)-\varphi\left(-x_{0}, \boldsymbol{x}\right)}{2 x_{0}} .
$$

$\chi_{k}$ is a test function and even with respect to the variable $x_{0}$. We can write now

$$
\begin{aligned}
\left(\Delta(x, s), \varphi\left(x_{0}, \boldsymbol{x}\right)\right)_{x_{0}} & =\int_{-\infty}^{+\infty} d x_{0}\left|x_{0}\right|\left(x^{2}\right)_{+}^{k} \frac{J_{k}\left(\sqrt{s x^{2}}\right)}{\left(\sqrt{s x^{2}}\right)^{k}} \chi_{k}\left(x_{0}, \boldsymbol{x}\right) \\
& =\int_{0}^{\infty} d s^{\prime} \int_{-\infty}^{+\infty} d x_{0}\left|x_{0}\right| \delta\left(x_{0}^{2}-|\boldsymbol{x}|^{2}-s\right)\left(s_{+}^{\prime}\right)^{k} \frac{J_{k}\left(\sqrt{s s^{\prime}}\right)}{\left(\sqrt{s s^{\prime}}\right)^{k}} \chi_{k}\left(x_{0}, \boldsymbol{x}\right) \\
& =\int_{0}^{\infty} d s^{\prime}\left(s^{\prime}\right)^{k} \frac{J_{k}\left(\sqrt{s s^{\prime}}\right)}{\left(\sqrt{s s^{\prime}}\right)^{k}} \cdot\left(\varepsilon\left(x_{0}\right) \delta^{k+1}\left(x^{2}-s\right), \varphi\left(x_{0}, \boldsymbol{x}\right)\right)_{x_{0}} .
\end{aligned}
$$

The last formula is valid for all $k=0,1,2, \ldots$.

If we choose $k$ sufficiently large, the function:

$$
s \leadsto\left(s_{+}^{\prime}\right)^{k} \frac{J_{k}\left(\sqrt{s s^{\prime}}\right)}{\left(\sqrt{s s^{\prime}}\right)^{k}} \quad\left(s^{\prime} \geqq 0\right)
$$

defines a test function for $h(x, \cdot)$ depending continuously on the parameter $s^{\prime} \geqq 0$. This function is a test function because it is analytic and:

$$
\left|\left(\frac{\partial}{\partial s}\right)^{n} \frac{J_{k}\left(\sqrt{s s^{\prime}}\right)}{\left(\sqrt{s s^{\prime}}\right)^{k}}\right|=o\left(s^{-\frac{1}{2}}\right) \quad \text { for } \quad s \rightarrow+\infty .
$$

The following function of $\boldsymbol{x}$ and $s^{\prime}$ is therefore well defined:

$$
g(\boldsymbol{x}, s):=\left(s_{+}^{\prime}\right)^{k} \cdot\left(h(\boldsymbol{x}, s), \frac{J_{k}\left(\sqrt{s s^{\prime}}\right)}{\left(\sqrt{s s^{\prime}}\right)^{k}}\right)_{s^{\prime}} . \quad\left(s^{\prime} \geqq 0\right)
$$

Our assumptions for $h(\boldsymbol{x}, s)$ tell us that this function has a compact spectrum and it depends continuously on $s^{\prime}$. Thus we have the equation:

$$
(T(x), \varphi(x))=\int_{0}^{\infty} d s^{\prime}\left(g\left(x, s^{\prime}\right) \varepsilon\left(x_{0}\right) \delta^{k+1}\left(x^{2}-s\right), \varphi(x)\right) .
$$

This is our representation (3). 


\title{
References
}

1. Magg, M.: Begründung der Lichtkegeldominanz für eine gewisse Klasse von Strukturfunktionen im Bjorken Limes. Dissertation der Mathematisch-Naturwissenschaftlichen Fakultät der RWTH Aachen, 1973

2. Brandt, R.A.: Phys. Rev. Letters 22, 1149 (1969); Phys. Rev. Letters 23, 1260 (1969); Phys. Rev. D1, 2808 (1970)

Joffe, B. L.: Phys. Lett. 30B, 123 (1969)

Brown,L.S.: Causality in electroproduction at high energy. In: Mahanthappa, K.T., Brittin,W.E. (Eds.): Lectures in theoretical physics, Vol. XII-B. New York: Gordon and Breach, 1971

Jackiw, R., van Royen, R., West, G. B.: Phys. Rev. D2, 2473 (1970)

Leutwyler,H., Stern, J.: Nucl. Phys. B20, 77 (1970)

3. Ryan, C.: Asymptotic regions in momentum space and light cone in configuration space. Dublin: preprint (1971)

4. Jaffe, R.L.: Phys. Rev. D6, 716 (1972)

Bhaumik, D., Greenberg, O.W.: Phys. Rev. D7, 3136 (1973)

Schwarz, F.: Fortschr. Physik 21, 839 (1973)

5. Bogoliubov, N.N., Takhelidze,A.N., Vladimirov,V.S.: Theor. Math. Phys. 12, 839 (1972)

6. Brüning, E., Stichel, P.: Commun. math. Phys. 36, 137 (1974)

7. Schwartz, L.: Theorie des distribution. Paris: Hermann 1966

8. Lighthill, M.J.: Fourier analysis and generalized functions. Cambridge University Press, 1958

9. Bogoliubov, N. N., Vladimirov, V.S.: Nauchn. Dokl. Vysshei Shkoly 3, 26 (1958)

10. Leutwyler, H., Otterson, P.: Theoretical problems in deep inelastic scattering (1972). In: Gatto, R.(Ed.): Scale and conformal symmetry in hadron physics. New York: John Wiley and Sons Inc. 1973

11. Drell, S. D., Walecka, J.: Ann. Phys. (N.Y.) 28, 18 (1964)

12. Meyer,J.W., Suura, H.: Phys. Rev. 160, 1366 (1967)

13. Wightman,A.S.: Analytic functions of several complex variables. In: De Witt-Omnes (Ed.): Relations de dispersion et particules elementaires. Paris: Hermann 1960

14. Gel'fand, J.M., Shilov, G. E.: Generalized functions, Vol. 1 and 2. New York: Academic Press 1964 and 1968

15. See especially Chapter VI, $\S 8$ of the book mentioned in [7]

16. Zemanian,A.H.: Distribution theory and transform analysis. New York: Mc Graw Hill 1965; see especially p. 94

17. Frishman, Y.: Talk given at the Second International Conference on Fundamental Interactions at High Energy, Coral Gables, January 1971: Brandt, R. A., Preparata, G.: Nucl. Phys. B27, 541 (1971)

18. Stichel, P.: Lectures given at the ecole internationale de la physique des particules elementaires. Basko Polje-Makarska (Yougoslavie), 1971

19. See Paragraph 45 in: Donoghue,W.F., Jr.: Distributions and fourier transforms. New York: Academic Press 1969

Communicated by R. Haag

\author{
M. Magg \\ Institut für Theoretische Physik \\ Technische Hochschule Aachen \\ D-5100 Aachen \\ Schinkelstraße 2/IV . \\ Federal Republic of Germany
}

